\title{
www.geotechnique.org et la géotechnique sur Internet
}

Ph. MESTAT

Laboratoire central des ponts et chaussées 58, boulevard Lefebvre 75732 Paris Cedex 15 Philippe.Mestat@lcpc.fr

\section{BERNHARDT}

Terrasol

Immeuble Helios

72, avenue Pasteur 93108 Montreuil Cedex v.bernhardt@terrasol.com

Dans les milieux scientifiques et techniques, une part de plus en plus importante de l'information et de la communication passe désormaís par l'Internet. Les professions s'organisent et se doivent d'être représentées sur le réseau des réseaux. A l'initiative du CFMS et en collaboration avec le CFMR et le CFGl, un site Internet www.geotechnique.org a été créé et est accessible depuis mai 2000. Cet article présente, dans une première partie, les objectifs qui ont guidé le groupe de travail ; il détaille ensuite plus particulièrement le site du CFMS. L'une des ambitions de ce site est d'être un point d'entrée pour celui, ingénieur ou chercheur, qui recherche des informations sur l'Internet dans le domaine de la géotechnique : documents, logiciels, listes de liens, bases de données, etc. On propose en complément, dans une seconde partie, un rapide panorama des ressources documentaires en géotechnique disponibles sur l'Internet et quelques conseils et recommandations pour naviguer avec efficacité.

Mots-clés : géotechnique, Internet, mécanique des sols, mécanique des roches, géologie de l'ingénieur, bibliographie, documentation, ressources, recherche.

\section{www.geotechnique.org and the geotechnical Web}

NDLE: Les discussions sur cet article sont acceptées jusqu'au 1'r octobre 2001.
More and more scientific and technical information is now exchanged through Internet. In this regard, geotechnica encineering must be present on the World Wide Web. The French Society of Soli Mechanics (CFMS), in collaboration with the French Societies of Rock Mechanics and of Engineering Geology, created a site dedicated to geotechnical engineering (avallable since May 2000) entitled www:geotechnique.org. This article presents the objectives of the working group who developed the site. The article also describes the CFMS site in more details. The main ambition of the site is to provide an access to engineers and researchers involved in geotechnical engineering, and to present papers, books, links, conferences, codes, database, etc. A review of on-fine information and some useful suggestions to navigate the internet are also provided.

Key words : geotechnical engineering, soll mechanics, rock mechanics, engineering geology, bibliography, documentation, resources, research. 


\section{Introduction}

LInternet connait depuis le début des années 90 une croissance exponentielle du nombre d'utilisateurs, de serveurs et de pages Web. En octobre 2000, le nombre total d'internautes était évalué à 360 millions. Rien qu'en France, certains analystes estiment que le nombre d'internautes atteindra 10,9 millions à la fin de l'année 2000. Selon Netvalue, les internautes français se connectent en moyenne 10,6 jours par mois et passent 5,5 heures sur le réseau des réseaux. Ils sont $48,1 \%$ à se connecter au travail, $34,9 \%$ à domicile et $39,9 \%$ dans un autre lieu.

C'est dans la communauté scientifique et technique que l'apport de l'Internet est le plus significatif. Son usage se généralise parmi les chercheurs, dans les grandes entreprises, et plus récemment dans les PME/PMI, gráce à l'apport formidable des messageries. des protocoles d'échanges de données et de la mise en réseau des informations. Alors que les congrès et les possibilités de publication se multiplient, la connaissance, la maitrise des flux d'informations et leur évaluation sont devenues de formidables enjeux. C'est dans ce contexte qu'à l'initiative du CFMS et en collaboration avec le CFMR et le CFGI, le site Internet commun aux trois comités www.geotechnique.org a été créé, pour informer la communauté scientifique et technique des activités des comités et offrir des services documentaires aux membres des comités et aux différents acteurs du secteur du génie civil.

LInternet peut être vu comme une gigantesque base de données et de services, de textes, d'images et de sons que chacun alimente et dans laquelle chacun cherche, gratuitement ou non. Le nombre de services offerts est impressionnant. En dehors des services pratiques, l'intérêt est évident à la fois en termes de communications (messageries), de transfert de données et de recherches d'informations (bibliographie, veille). C'est un outil facile d'accès et extraordinairement puissant pour peu de maitriser les bases essentielles nécessaires pour entamer les recherches. Cet intérêt est malheureusement perturbé par la profusion des informations et leur plus ou moins grande fiabilité. Le problème n'est pas seulement de trouver l'information, il est aussi de l'apprécier à sa juste valeur. Les sites des Sociétés savantes et des grands organismes de recherche peuvent alors jouer un rôle de conseils en recommandant des pages de liens sélectionnés pour leur sérieux et leur qualité technique ou scientifique.

TAatsau I Nombre de pages Web repérées correspondant à quelques mots-clés de la géotechnique (moteur Alta Vista, recherche avancée effectuée en octobre 2000).

Number of webpages corresponding to a few geotechnical keywords (advanced search on Altavista, October 2000).

\begin{tabular}{|l|r|}
\hline \multicolumn{1}{|c|}{ Mots-clés } & Nombre de pages \\
\hline Géotechnique & 2941 \\
Mécanique des sols & 1498 \\
Mécanique des roches & 721 \\
Géologie de l'ingénieur & 459 \\
Géologie & 27452 \\
Geotechnics & 148805 \\
Soil mechanics & 19301 \\
Rock mechanics & 14765 \\
Engineering geology & 24080 \\
Geology & 1023644
\end{tabular}

Comme pour les autres disciplines, le nombre de sites dédiés à la géotechnique est en constante augmentation. On peut estimer que, depuis trois ans, le nombre de pages consacrées à la géotechnique a été multiplié par environ 150 ! L'utilisation d'un moteur de recherche permet d'avoir une idée de cette profusion. A titre d'exemple, le tableau I illustre le nombre de pages web repérées par le moteur Alta Vista pour certains mots-clés reliés à la géotechnique et à la géologie de l'ingénieur.

Nota: Cet article a été rédigé en octobre 2000 et les informations fournies (chiffres, liens, copies d'écran, etc.) auront done probablement évolué au moment de sa pubiication. Nous vous invitons notamment á visiter le site www.geotechnique.org pour visualiser les rubriques à jour.

\section{2}

\section{Le site Internet du CFMS}

\section{1}

\section{Pour la petite histoire}

En septembre 1999, un groupe de travail a été formé au sein du CFMS et son animation confiée à Philippe Mestat. Une première réunion a permis de définir les principales rubriques et de nommer, pour chacune, un responsable chargé de veiller à sa création puis à son actualisation. Une première maquette a été réalisée par Valérie Bernhardit à titre bénévole et validée au cours d'une seconde réunion en mars 2000. C'est à cette occasion qu'il a été décidé de proposer au CFMR et au CFGI de créer une page commune dédiée à la géotechnique et à la géologie de l'ingénieur françaises, à partir de laquelle un internaute pourra se diriger vers le site de chaque comité ou vers celui de la Revue Francaise de Géotechnique (RFG), revue commune au CFMR, au CFGI et au CFMS. Les trois comités et la RFG se sont ainsi mis d'accord sur la création du site www.geotechnique.org. Le nom du domaine a été déposé au mois de mars 2000. La page d’accueil est présentée sur la figure 1.

A partir de cette page intitulée « Site officiel des comités français de la géotechnique et de la géologie de l'ingénieur »), on peut se diriger vers la RFG ou vers l'un des trois comités (CFMS, CFMR, CFGl) en cliquant soit sur leur nom, soit sur leur logo. Selon le choix de l'internaute, une nouvelle page d'accueil s'affichera à l'écran. Chaque comité est naturellement responsable du contenu de son site, tout comme la Rédaction en chef de la RFG est responsable du contenu des pages de son site.

Pour l'organisation matérielle, le groupe de travail du CFMS a accueilli les représentants du CFMR et du CFGI et les maquettes respectives ont également été réalisées par Valérie Bernhardt. Celles-ci sont basées sur la même structure que celle du CFMS et les pages d'accueil ont la même allure ; chaque comité ayant choisi « sa » couleur dominante. L'ensemble du site est conçu pour être visualisé sur un écran de résolution 800 × 600 au minimum. Il a été mis en ligne au mois de mai 2000. Peu de temps après, le site du CFMS a été présenté officiellement lors de l'assemblée générale de l'association. C'est actuellement le site dont la construction est la plus avancée. Il aura fallu à peu près un an entre la décision de créer un site et la mise en ligne de la première version. C'est ce site qui est plus particulièrement décrit dans ce qui suit. 

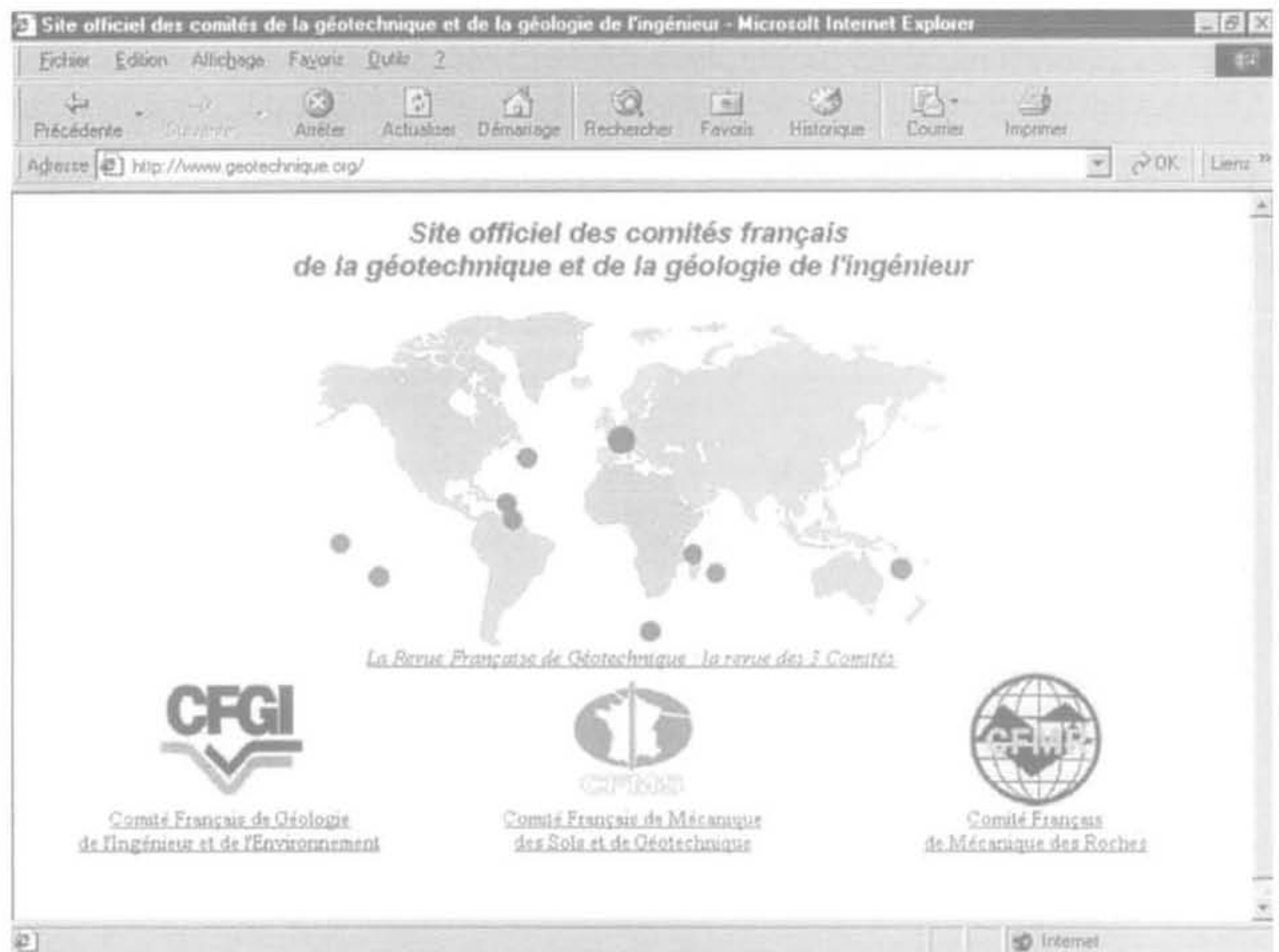

[]

FIG.1 Copie d'écran de la page d'accueil du site www.geotechnique.org (rẻalisée en octobre 2000).

(Les points inscrits sur la carte du monde représentent les territoires français.)

Screenshot of the www.geotechnique.org homepage. (Red points displayed on the world map represent French territories).

2.2

\section{Objectifs du site Internet du CFMS}

Le site Internet du CFMS est un outil de communication pour informer :

- plus rapidement les membres du CFMS et les personnes intéressées par la Mécanique des sols (actualités scientifiques et techniques, manifestations du CFMS, groupes de travail, annonces diverses, etc.) :

- plus largement. La diffusion du Bulletin du CFMS et de la Lettre de la géotechnique ne s'adressait jusqu'ici qu'aux membres. Désormais, avec l'Internet, le CFMS présente ses activités et s'adresse à tous les internautes. Cela concerne en particulier les jeunes géotechniciens et les étudiants

- plus efficacement en offrant de nouveaux services à ses membres et aux différents acteurs du secteur du génie civil. Le site du CFMS doit être vu aussi comme un point d'entrée et un guide pour la recherche d'informations sur l'Internet (textes, images, logiciels, services, produits). Une liste de liens intéressants et commentés vers des sites francophones ou anglophones est ainsi proposée.

Le site du CFMS devrait constituer à terme un relais entre les géotechniciens francophones: les uns pourront alimenter les différentes rubriques du site et les autres découvrir périodiquement de nouvelles informations et de nouveaux services. Cela suppose bien sûr que les propositions d'informations seront nombreuses et régulières. L'enjeu est de construire et de faire vivre un site d'échanges au profit de tous les géotechniciens francophones.

\section{3}

\section{Le contenu du site du CFMS}

Le site du CFMS est organisé autour de six rubriques principales :

- Page d'accueil :

- Informations générales :

- Manifestations :

- Actualité géotechnique ;

- Documentation :

- Liens.

\section{arain}

\section{Accueil et mise en page du site}

La page d'accueil est composée de trois éléments (Fig. 2) :

- le bandeau supérieur qui contient notamment le logo de l'association, la date de la dernière mise à jour et le compteur des visiteurs. Ce bandeau est fixe et reste visible pendant toute la visite du site ;

- le navigateur à gauche de l'écran qui correspond au sommaire du site. Il est également constamment visible, mais il évolue en fonction des rubriques sélec- 


\begin{tabular}{|c|c|c|c|c|c|c|c|c|c|c|}
\hline Fechier & Eótion & Alichoge & Fixula & givet ? & & & & & & \\
\hline 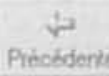 & 2. & interte & Aneter & Actusised & Defmarioge & Pecheicher & $\frac{\text { Farois }}{\text { Far }}$ & $\begin{array}{c}\text { Historigue } \\
\text { Hes }\end{array}$ & $\frac{\text { Courier }}{\text { Co }}$ & Imcrimer \\
\hline
\end{tabular}

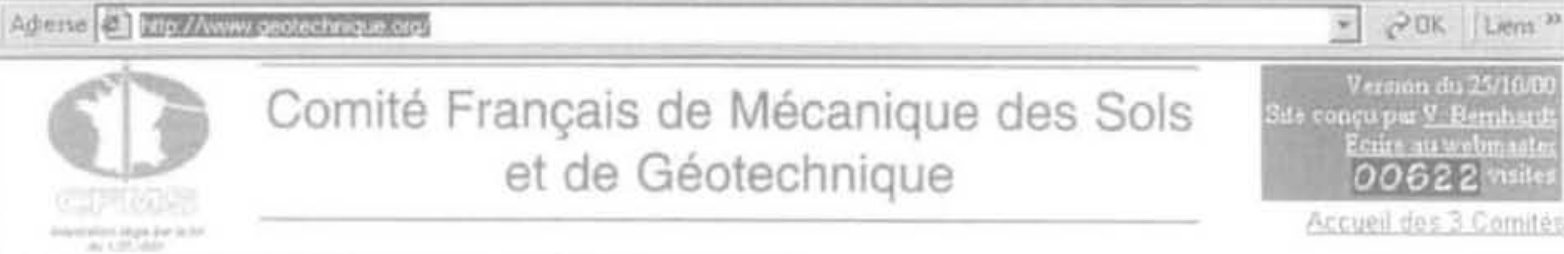

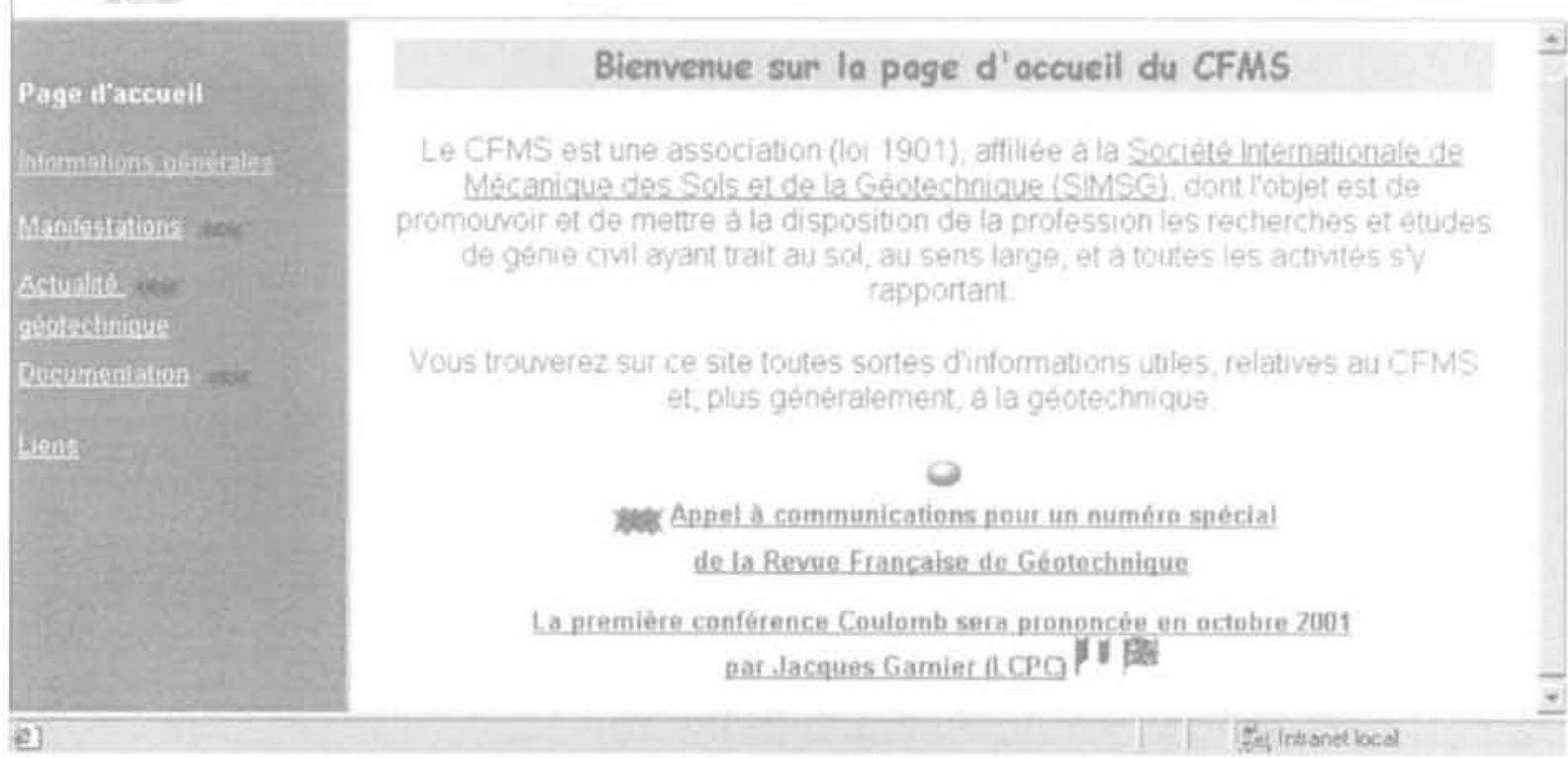

F16.2 Copie d'écran de la page d'accueil du site du CFMS (réalisée en octobre 2000). Screenshot of the CFMS homepage, dated October 2000.

tionnées par le visiteur. Lorsqu'elle est « cliquée», une rubrique se déplie et propose des sous-rubriques (Figs. 3 à 5). Les nouveautés sont signalées par l'incrustation « new » en rouge :

- la page d'accueil elle-mème, qui comprend une présentation en quelques lignes du CFMS pour les visiteurs ne connaissant pas l'association, ainsi que les dernières informations générales susceptibles d'intéresser les membres : appel à communications, annonce de l'assemblée générale, élections, nominations, etc.

\section{Informations générales}

Cette rubrique est destinée à présenter le CFMS, son statut, ses objectifs et son organisation. Les sousrubriques disponibles actuellement sont les suivantes : - présentation (principaux objectifs de l'association); - affiliation à la SIMSG (informations sur la Société internationale de mécanique des sols et de géotechnique, lien vers son site international:

www.issmge.org) :

- statuts (texte en ligne des statuts du CFMS) ;

- conseil et commission technique (composition du bureau, du conseil et de la commission technique ; cette page est actualisée après chaque élection) ;
- francophonie (présentation de la commission pour la francophonie et de ses activités) ;

- commissions techniques internationales (liste des commissions techniques internationales et des commissions techniques européennes : numéros, noms, membres français, président, liens vers les sites existants, Fig. 3) :

- bulletin d'adhésion (téléchargement du bulletin d'adhésion au CFMS, à remplir et renvoyer au secrétariat du CFMS. Les tarifs d'adhésion sont rappelés sur la page);

- contacts (coordonnées du secrétariat, adresses électroniques du président du CFMS, du président de la commission technique et des responsables de chaque rubrique du site. C'est à ces derniers qu'il faut envoyer vos remarques, idées et informations concernant le site du CFMS).

\section{Cani3 \\ Manifestations}

Cette rubrique annonce l'ensemble des manifestations (congrès, colloques, conférences, formations continues, etc.) concernant la géotechnique, en France comme à l'étranger, qui a été signalé au secrétariat du CFMS. La mise à jour de cette rubrique est prévue tous les deux mois environ. 
I Echier Edition Asfichoge Facois Quith ?

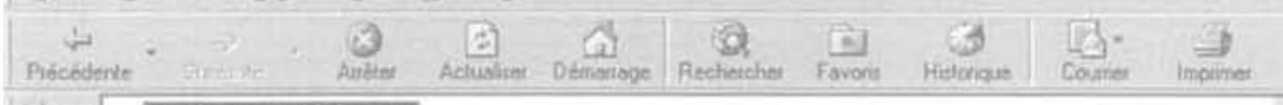

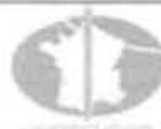

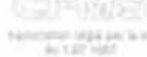

\section{Comité Français de Mécanique des Sols} et de Géotechnique

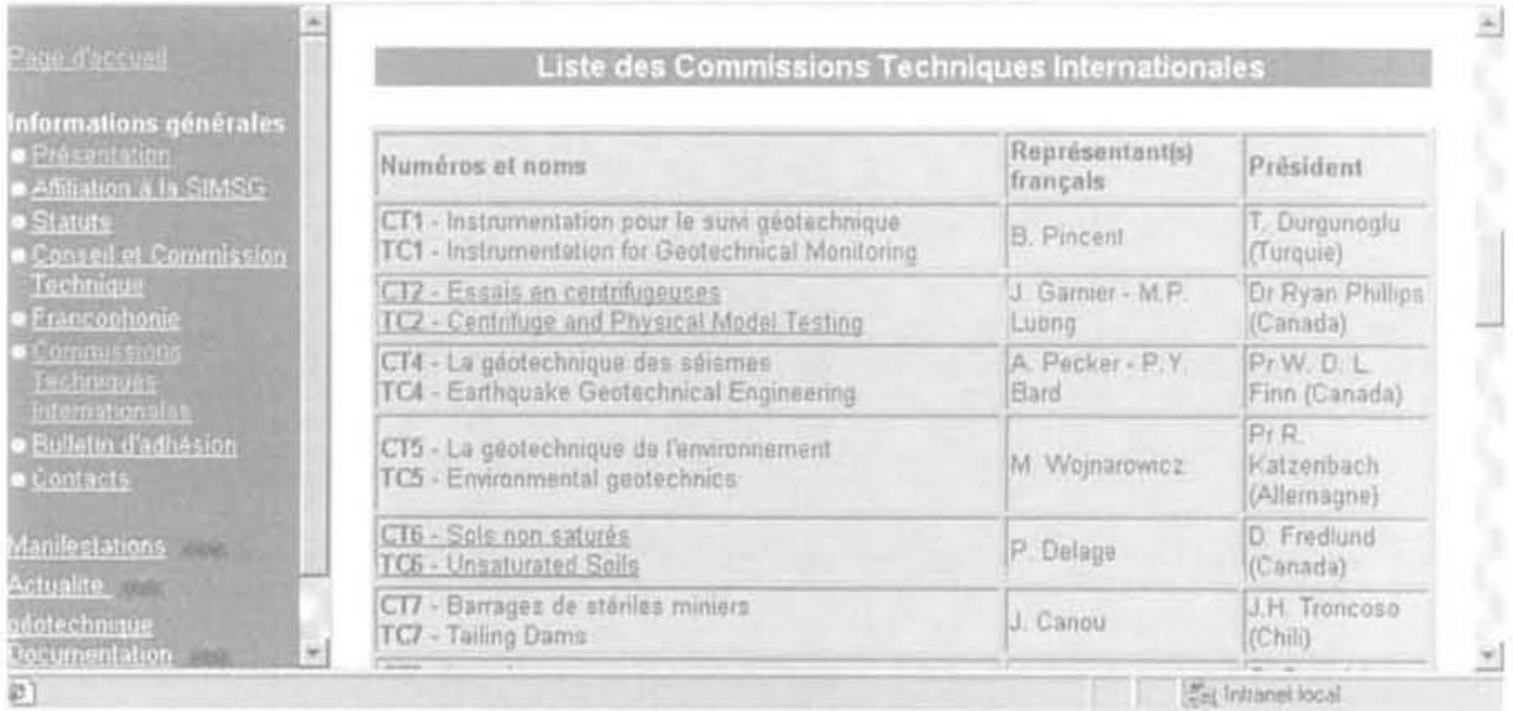

FIG.3 Copie d'écran de la page “ Commissions techniques internationales » (réalisée en octobre 2000).

Screenshat of the page « Commissions techniques internationales $x$ (international technical committees), dated October 2000

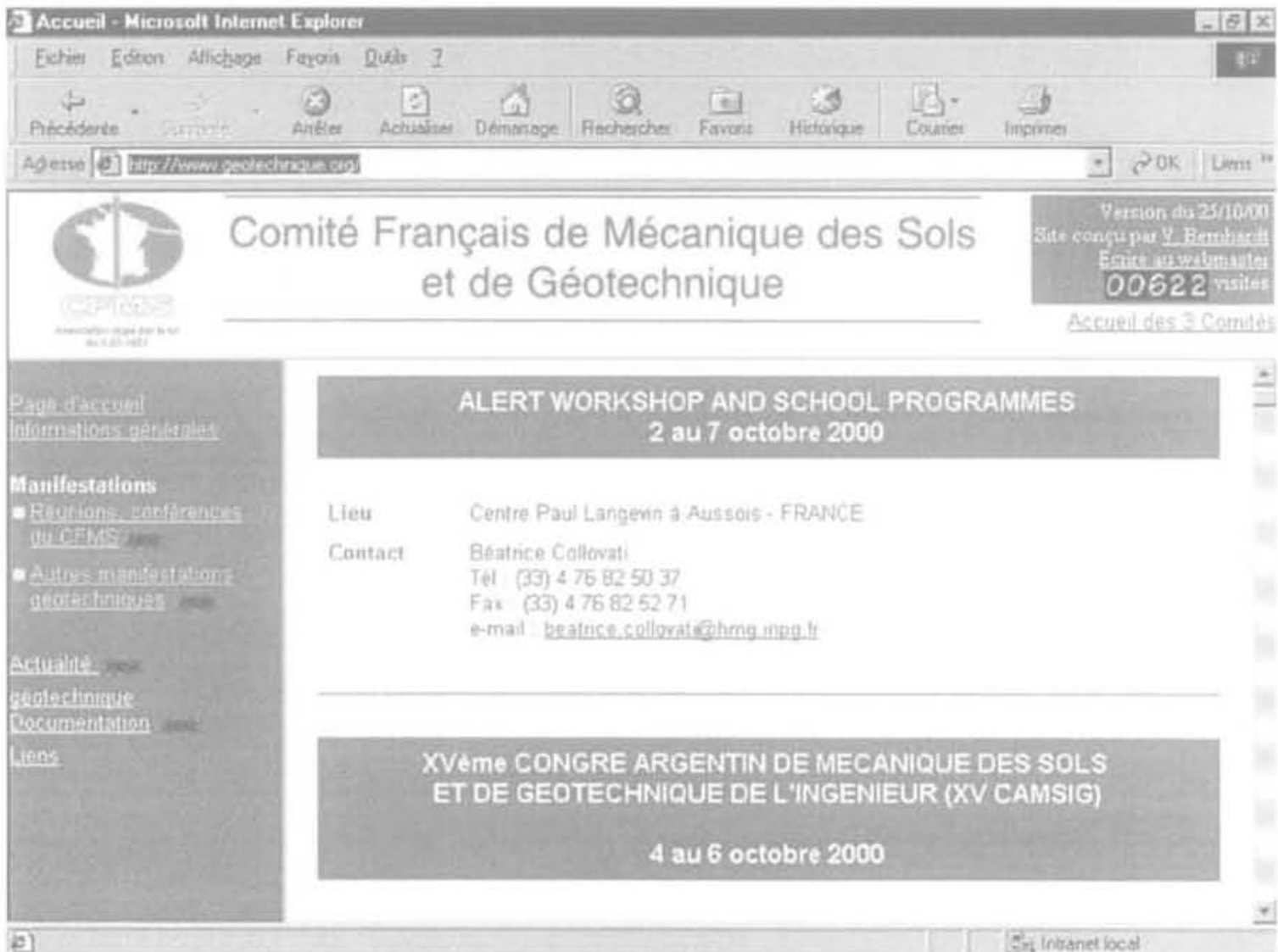

FG.4 Copie d'écran de la page " Autres manifestations géotechniques » (réalisée en octobre 2000).

Screenshot of the page «Autres manifestations géotechniques n) (other geotechnical events), dated October 2000. 
On a distingué deux sous-rubriques :

- réunions, conférences du CFMS, rubrique qui abrite le programme, généralement détaillé, des prochaines manifestations organisées par le CFMS (journées techniques, assemblées générales, etc.) :

- autres manifestations géotechniques, dans laquelle le visiteur retrouve la liste des autres manifestations prévues dans le domaine de la géotechnique, en France comme à l'étranger. Pour chaque manifestation, toutes les informations disponibles sont indiquées : date, lieu, thème, coordonnées de l'organisateur et, éventuellement, la date limite pour la présentation de résumés et des communications, etc. Le début de la page est affiché en copie d'écran sur la figure 4.

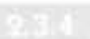

\section{Actualité géotechnique}

Cette rubrique d'actualité doit proposer des informations nouvelles et vérifiées dans le domaine de la géotechnique. Pour cela, le CFMS appelle ses membres à alimenter cette rubrique en fournissant des informations récentes au secrétariat du CFMS ou au responsable de cette rubrique (Michel Gambin). C'est notamment le cas pour les comités techniques européens et internationaux. L'objectif est de mettre à jour la rubrique tous les deux mois environ.

On a créé actuellement cinq sous-rubriques :

- appels à communication. Le visiteur y trouvera la liste des manifestations à venir pour lesquelles des appels à communication sont en cours. Cette page fournit également la date, le lieu, le thème, les coordonnées des organisateurs ainsi que les modalités pour présenter une communication :

- communications acceptées. Cette page est destinée à accueillir les informations concernant les communications françaises acceptées à des congrès ou colloques organisés par la Société internationale de mécanique des sols et de géotechnique (SIMSG). Aujourd'hui, la page contient la liste des communications retenues pour le prochain congrès d'Istanbul. A terme, le visiteur pourra consulter les résumés des communications acceptées

- actualité de la francophonie. Cette page est en cours de construction :

- actualité des groupes de travail du CFMS. Pour chacun, sont donnés les objectifs, la composition du groupe et l'état d'avancement des travaux;

- actualité des TC internationaux. Cette page est également en cours de construction : elle devrait être alimentée par les participants français aux différents comités techniques internationaux.

\section{3:-3: \\ Documentation}

Cette rubrique a pour objectif de mettre à la disposition des visiteurs du site un maximum de documentation dans le domaine de la géotechnique. Les sousrubriques suivantes sont accessibles :

- la Revue française de géotechnique. Cette page est commentée ci-après ;

- la Lettre de la géotechnique. Cette page propose au visiteur de télécharger au format pdf les derniers numéros de la Lettre de la Géotechnique. Comme il faut disposer du logiciel Acrobat Reader pour lire ces fichiers, la page propose un lien pour télécharger ce logiciel gratuit ;

- exposés des conférences du CFMS. L'objectif est de proposer en ligne les textes, transparents ou diapositives présentés lors des journées ou demi-journées techniques du CFMS. Nous comptons sur les conférenciers pour nous fournir ces données sur un support informatique adéquat;

- bibliothèques, dans laquelle l'internaute découvrira une liste d'ouvrages de référence en langue française (mécanique des sols, hydraulique, dynamique, séismes, etc.). Des liens vers les sites des principaux éditeurs scientifiques sont également indiqués. Cette page sera complétée en fonction des suggestions des visiteurs :

- autres publications. Cette page est en cours dé construction; elle pourrait accueillir des résumés de thèses ou divers rapports accessibles en texte intégral.

\subsection{6.}

Liens

Cette dernière rubrique propose deux types d'informations :

- membres collectifs. Il s'agit de la liste de tous les membres collectifs adhérents du CFMS, avec leurs coordonnées. Prochainement (c'est peut-ètre déjà le cas au moment où cet article est publié), chaque membre collectif pourra disposer d'un lien direct vers son propre site ;

- liens externes. Cette rubrique est destinée à être un point de départ pour celui ou celle qui cherche des informations sur l'Internet dans le domaine de la géotechnique. Les liens proposés sont classés en différentes catégories : annuaires, bases de données, bibliothèques virtuelles, revues, sociétés savantes, etc. Pour chaque lien proposé, le visiteur trouvera un commentaire sur le site correspondant (Fig. 5).

\section{4}

\section{Perspectives de développement}

Le souci des responsables du site du CFMS est de fournir aux géotechniciens francophones un maximum d'informations utiles et mises à jour régulièrement. Disponible en ligne depuis le mois de mai 2000, la version du site présentée ici constitue une première étape. Naturellement, il sera possible de l'enrichir et d'élargir encore la gamme des services.

En complément des évolutions envisagées pour les rubriques existantes, nous pensons par exemple mettre en ligne l'annuaire des membres du CFMS. Ceci permettrait d'éviter une édition a papier $»$ et d'avoir une mise à jour réqulière, rapide et simple des coordonnées de chacun. Toutefois, pour des raisons légales, cela ne sera possible qu'après accord écrit de chaque membre. A cet effet, un questionnaire sera envoyé à tous les membres du CFMS.

Il est également envisagé de créer une version anglaise du site, avec notamment des informations utiles pour les internautes à la recherche d'éléments sur la géotechnique francophone. 


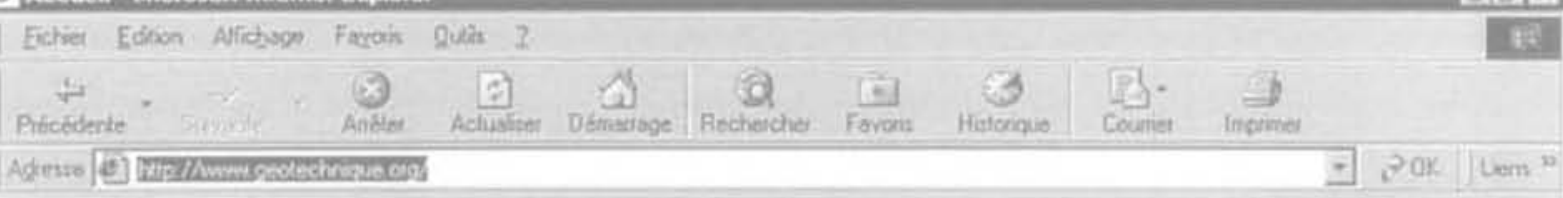

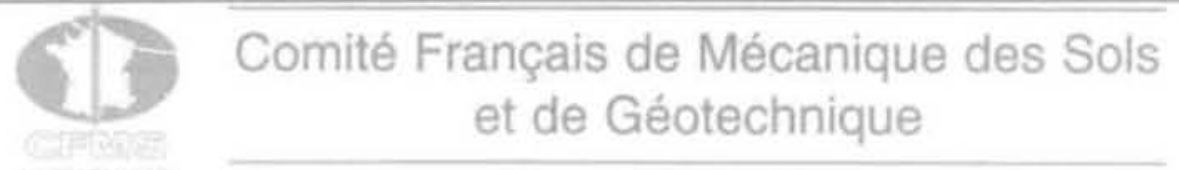

Yeraton dushion et de Géotechnique

Programmes de recherche européens dans fe domaine de la gêtufechnique Le rềseau NFCER Zes:

Ce stie présente le programme de recherche auropèan NECEF sur le developpement de ta modèlisation physique en centrifugeuse, en géotechnique de lenvionnament if soorganse autoul dine collaboration entre 11 latoratoires, danis 7 pays dEurope Un symposium intemational. Alargissant ce theme a tous ses aspects de lapproche expermentale, a ete organige i La Baule en mai 2000

Actualice de ta Geatechnique

Hagazine èlectronique de la génotechnique

Ce site propose un ensemble de rubrique dractualites des articles, des cas defutes, dea annonces de conferences, une liste des 10 melleurs sites de Geofechnique, des pages dannonces dmerses, des logiciels, des videtos, etc

Annonce de prochaines conférences

Liste des conférences dans le domaine de is Géotechnique Mise a jour régulietre

\section{Annuairens géstechniques}

Artresses des sncithis savantes

Ce site abrte un annuaire des sites des Societes Sarantes dans tous les domaines On trouvera en particulier les adresses des principales Sociétes Savantes du Genie Ciml el des Scrences de la Terre

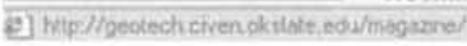
Fis Intranetioced

FiG.5 Copie d'écran de la page “Liens externes») (réalisée en octobre 2000). Screenshot of the page «Liens externes y (external links), dated October 2000.

L'actualisation régulière de l'ensemble des rubriques ne sera possible qu'avec la participation active de tous les intervenants français et francophones. Nous comptons sur eux pour nous transmettre les informations portées à leur connaissance, en particulier pour alimenter la rubrique « Actualité 1).

Par ailleurs, les opérations de référencement du site et des pages sont en cours. Le site du CFMS sera ainsi accessible à partir des principaux moteurs de recherche et annuaires disponibles sur I'Tnternet.

\section{3}

\section{La page de la Revue française de géotechnique}

Depuis la page d'accueil du site des trois comités, on peut accéder directement à la page consacrée à la Revue Française de Géotechnique. Cette page (dont une partie est visible sur la figure 6) est accessible également depuis la rubrique «Documentation $n$ du site du CFMS et, dans un proche avenir, des rubriques similaires des sites du CFMR et du CFGI.

Sur cette page sont présentés la composition du comité de lecture, les instructions relatives aux auteurs et les sommaires des derniers numéros. En cliquant sur le titre d'un article, l'internaute peut faire afficher les résumés en français et en anglais correspondants. D'autres services documentaires (gratuits ou payants) sont à l'étude en collaboration avec la rédaction de la
RFG et Ponts Formation Édition : liste des articles depuis le premier numéro de la revue, recherche par mots-clés, téléchargement d’article, etc.

\section{4}

\section{La géotechnique sur l'Internet}

L'un des grands intérêts de l'Internet est de pouvoir consulter des sources documentaires diverses et variées. Encore faut-il y accéder sans perdre trop de temps. A moins de connaitre parfaitement sa destination d'arrivée, la « navigation $»$ sur le Web est facilitée par les moteurs de recherche disponibles gratuitement. Malgré cette aide précieuse, trouver rapidement les informations que l'on cherche parmi plusieurs centaines de milliards de pages html n'est pas une chose aisée. Il y faut de la réflexion et de la méthode, de la rigueur et de la précision mais aussi quelques notions sur le fonctionnement du réseau et des moteurs de recherche. Cette seconde partie a ainsi pour objet de prodiguer quelques conseils et recommandations, issus d'une pratique régulière de l'Internet.

\section{1}

\section{Repérer des informations}

Connaître ses besoins en informations est essentiel pour initier une recherche. Selon ces besoins, l'approche de l'Internet et les sources documentaires à 


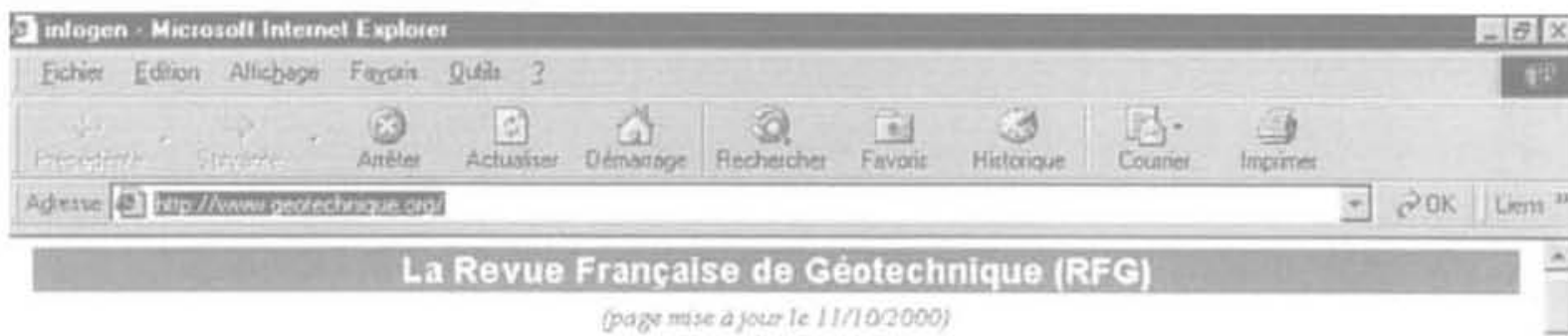

Appel à communications pour un numéro spécial

de la Revue Française de Géotechnique

REVUE

FRANCAISE

DE

GÉOTECHNIQUE

omité de lecture

Sommaires des demiers numerós

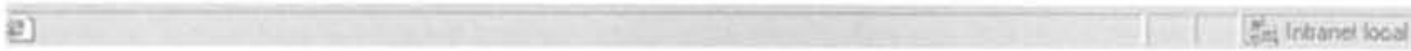

FIG.6 Copie d'écran de la page de la Revue française de géotechnique (réalisée en octobre 2000). Screenshot of the page of the a Revue française de géotechniquè, dated October 2000.

consulter peuvent être très différentes comme le seront les mots-clés pertinents à fournir aux moteurs de recherche. Le tableau Il décrit les ressources documentaires disponibles selon les catégories de besoin.

Trois grandes catégories d'outils existent pour aborder une recherche :

- les moteurs de recherche pour une requête directe sur le réseau au moyen d'un ensemble de mots-clés ; - les bibliothèques virtuelles pour une recherche à partir de thèmes et de sites présélectionnés :
- les sites de documentation des organismes de recherche, qui constituent aussi d'excellents points de départ (lorsqu'ils sont d'accès libre).

Les bibliothèques virtuelles et les services de documentation proposent fréquemment des listes de liens commentés vers d'autres sites spécialisés dans les mêmes domaines ou mieux un moteur de recherche propre à une discipline bien précise.

On peut citer encore quelques autres sources d'informations : les sites des Sociétés savantes, les

TABLEAUII Besoins en documentation et sites de l'Internet (Le Roux et al., 2000). Documentary needs and websites (Le Roux and Mestat, 2000).

\begin{tabular}{|c|c|c|}
\hline Type de besoin. & Exemples de recherche & Sources documeritaires sur l'Internet \\
\hline Informations générales & Encyclopédies, livres, cours, liste de liens & $\begin{array}{l}\text { Éditeurs, services DOC, bibliothèques, catalogues, sociè- } \\
\text { tés savantes, bases de données }\end{array}$ \\
\hline Informations détaillées & $\begin{array}{l}\text { Articles, préprints } \\
\text { Communications, résumés } \\
\text { Rapports techniques, normes, brevets }\end{array}$ & $\begin{array}{l}\text { Périodiques, bases de données spécialisées, journaux } \\
\text { électroniques } \\
\text { Conférences, éditeurs, bases de données spécialisées } \\
\text { Éditeurs, universités, sites gouvernementaux }\end{array}$ \\
\hline Renseignements ponctuels & $\begin{array}{l}\text { Adresses, listes d'organismes, de produits, } \\
\text { de logiciels, de chercheurs, Liste de cours, } \\
\text { de formations continues, etc. } \\
\text { Données physiques, images, sons }\end{array}$ & $\begin{array}{l}\text { Annuaires (pages jaunes), sociétés savantes, } \\
\text { associations professionnelles, sites des universités } \\
\text { et des écoles (annuaires et organigrammes) } \\
\text { Bases de données spécialisées. }\end{array}$ \\
\hline Informations récentes & $\begin{array}{l}\text { Nouveautés, informations sur les congrès } \\
\text { à venir }\end{array}$ & $\begin{array}{l}\text { Périodiques, sociétés savantes, sites des conférences, } \\
\text { news }\end{array}$ \\
\hline Veille technologique & Sommaires de revues, lettres spécialisèes & Éditeurs, sites de liens avec les périodiques, news \\
\hline
\end{tabular}


bases de données bibliographiques gratuites, les journaux électroniques...

Quel que soit l'outil finalement retenu pour démarrer la recherche, celle-ci aboutit à un moment ou un autre à une requête qu'il convient de formuler de manière appropriée. Cela demande un peu d'entraînement et de méthode pour choisir et hiérarchiser les mots-clés sélectionnés.

Par ailleurs, il faut savoir profiter pleinement de I'une des plus extraordinaires propriétés de ces réseaux, à savoir les liens établis entre sites qui permettent d'élargir les recherches et de rebondir tout en restant normalement au plus près de son sujet. De fil en aiguille, au risque parfois de se perdre, on peut accéder aux informations souhaitées.

\section{2}

\section{Utiliser un moteur de recherche}

Pour une recherche directe d'informations, la connaissance du fonctionnement d'un ou de plusieurs moteurs de recherche est fortement recommandée pour prendre un bon départ. De nombreux moteurs de recherche par mots-clés existent aujourd'hui et chacun explore le réseau de manière différente. Ce sont de gigantesques bases de données constituées automatiquement grâce à des « robots » qui inspectent à intervalles réguliers les serveurs déclarés sur l'Internet. Les documents localisés font l'objet d'une indexation qui peut porter sur le titre, l'entête des documents, quelques lignes, le premier paragraphe ou sur les documents complets. Par exemple, le moteur Alta Vista indique que son robot Scooter actualise l'indexation de plus de 350 millions de sites chaque semaine. Ces moteurs rendent de grands services à condition d'être bien utilisés et de trier les informations obtenues.

La masse de données recueillies est souvent considérable, et on n'exploite raisonnablement que les premières dizaines de références. C'est un des inconvénients actuels que de ne pouvoir poser l'interrogation qu'une seule fois. Affiner les recherches par des tris successifs est actuellement impossible. On ne peut qu'affiner l'interrogation en repartant de zéro ou presque, au risque de perdre de l'information. Il est donc fondamental de bien sélectionner ces mots-clés et de les hiérarchiser comme il faut dans l'équation logique qui sera soumise au moteur de recherche. Cette équation combine ces mots-clés avec un ou des opérateurs booléens ( ( $\mathrm{AND}$ ), « OR », ( $\mathrm{AND}$ NOT $)$ et ( NEAR $»)$. Le moteur décode cette équation, la transforme en critères de recherche et, assez rapidement eu égard aux milliards de pages existantes, affiche à l'écran une liste d'adresses de sites ou une liste de documents en hypertexte. A côté de chaque adresse se trouvent l'intitulé du site ou du document repéré et une ou deux lignes de texte pouvant éclairer sur le contenu et l'intérêt du site ou du document. L'ordre d'apparition des informations dépend du classement effectué par l'algorithme de tri utilisé par le moteur de recherche. Selon l'algorithme, les informations peuvent être classées différemment. On peut obtenir en tête de liste (d'après Jarcly, 1999): - les documents contenant tous les mots-clés demandés :

- ceux les contenant dans le titre ou au début du texte; - ceux pour lesquels les critères de recherche sont proches:

- ceux où les critères sont présents le plus grand nombre de fois.

Cette liste dépend aussi de l'ordre dans lequel les mots-clés ont été introduits dans l'équation logique et de la pondération opérée par l'algorithme de recherche. Si la combinaison proposée est peu précise ou peu pertinente, l'information récoltée sera considérable. Inversement, si la combinaison est trop précise ou trop restrictive, le moteur peut ne rien trouver ou passer à côté de sites essentiels car leur « référencement » (ou indexation) a été réalisé à un niveau plus général. Il y a un certain équilibre à trouver. La meilleure manière d'apprendre est encore de pratiquer et de faire l'expérience d'une recherche sur un sujet que l'on maitrise bien. C'est en pratiquant que l'on devient convaincu... et que l'on repère vite le moteur et les sites de départ qui conviennent à chacun (et qu'il est recommandé, pour gagner du temps, de placer dans ses « favoris $\gg)$. L'internaute a ses habitudes ; il suffit qu'elles soient bonnes.

Le moteur Alta Vista semble être le plus exhaustif. Tous les mots sur chaque page Web sont indexés. D'autres moteurs sont plus sélectifs. Par exemple, le moteur Yahoo évalue chaque site ou chaque document au préalable. Les résultats de la recherche sont classés par thèmes ou rubriques. Cela permet de démarrer une nouvelle recherche à partir des thèmes sélectionnés par le moteur et non de repartir sur tout le réseau.

Le logiciel gratuit Copernic, que l'on peut télécharger à l'adresse www.copernic.com/fr, permet notamment de consulter plusieurs moteurs de recherche simultanément et de stocker les résultats de plusieurs requêtes. C'est un outil très pratique et efficace.

Le tableau III énumère quelques conseils pour soumettre une équation logique au moteur de votre choix.

\section{TABLEAU III Conseils pour écrire une équation logique destinée à un moteur de recherche.} Advices to write a websearch request.

\footnotetext{
1. Délimiter le sujet de la recherche

2. Choisir la langue de travail (anglais ou français)

3. Définir un à trois mots-clés généraux (comme géotechnique, mécanique des sols, mécanique des roches, génie pétrolier, fondations, etc.)

4. Définir un ou plusieurs thèmes et y rattacher des mots-clés spécialisés (noms propres, noms communs, sigles, abréviations ou chiffres)

5. Sélectionner un moteur de recherche

6. Combiner un mot-clef gẻnéral avec deux ou trois mots-clés spécialisés dans une équation logique

7. Ajouter éventuellement à l'équation un ou deux mots-clés spécifiques de l'Internet cornme ressource ou resource, site, guide, library ou librairie, database ou databank, etc.

8. Penser aux variantes orthographiques (notamment aux orthographes anglaises et américaines : par exemple, colour et color, modelling et modeling, etc.) et aux synonymes

9. Exploiter les trente premiers résultats fournis par le moteur

10. S'il n'y a pas de sites intéressants, recommencer en combinant d’autres mots-clés spécialisés.
} 
A partir des premières adresses obtenues, on peut redéfinir le schéma de recherche ou se laisser guider par les connexions et aller de site en site à la découverte d'informations.

La recherche directe par mots-clés n'est donc pas toujours la plus pertinente. Néanmoins, cela vaut toujours la peine d'essayer. Si les résultats ne sont pas concluants ou trop abondants, il vaut mieux alors se tourner vers une bibliothèque virtuelle ou un site documentaire spécialisé. Si ces sites ne sont pas déjà connus de l'internaute, il aura pu découvrir leur adresse au cours de ses requêtes directes ou en se dirigeant sur des sites familiers comme maintenant celui du CFMS.

\section{3}

\section{Surfer avec les bibliothèques virtuelles}

L'autre manière de trouver de l'information dans une discipline bien identifiée consiste à se brancher sur les bibliothèques virtuelles existantes. Celles-ci sont de mieux en mieux conçues, couvrent des domaines assez larges et ont généralement procédé à des sélections dans les liens qu'elles proposent et gèrent. Ce sont en fait des catalogues commentés dans lesquels les sites sont classés par spécialités ou disciplines scientifiques et techniques. Elles aident l'internaute à arriver sans trop de détours à des serveurs susceptibles de l'intéresser et d'approfondir sa recherche.
La base de données des bibliothèques virtuelles existe (http://conbio.rice.edu/vl/database/) ; elles y sont classées par thèmes et par ordre alphabétique. On trouve ainsi facilement le thème "géotechnique » (geotechnical engineering) parmi beaucoup d'autres. Cela permet entre autre d'accéder rapidement aux sites de tous les départements de géotechnique des centres techniques et des universités (classés par continent puis par pays). Le tableau IV regroupe les adresses de quelques sites d'un grand intérêt pour la géotechnique. Pour le génie civil au seris large, on conseille notamment le site remarquable de la bibliothèque de l'université technique de Lunds en Suède (www.tenlinks.com/civil) et celui de la bibliothèque virtuelle d'Édimbourg (www.eevl.ac.uk), qui propose un moteur de recherche performant. Certains des liens cités ont été introduits dans la rubrique "Liens externes n du site du C.FMS

Signalons également l'excellent site maintenu par I'Ecole universitaire d'ingénieurs de Lille (www.eudil.fr/eudil/genciv/index.htm) qui offre plus de 250 liens concernant les formations, les centres de recherche, les métiers, les entreprises (groupes de BTP, bureaux d'études), les institutions (ministères, DDE, fédérations, syndicats, associations), les grands ouvrages (banques d'images), la documentation (bibliothèques, éditeurs, revues), les forums de discussion, les meilleurs sites étrangers, les cours en ligne, les manifestations, etc.

TABLEAUIV Quelques sites généraux pour la géotechnique (tableau établi en octobre 2000). A few general websites relating to geotechnique. Table dated October 2000.

\section{Description du site}

Adresse sur l'Internet

http://www.tenlinks.com/civil

Bibliothèque de l'université de Lunds (plus de 1000 liens avec les principaux sites du génie civil)

Bibliothèque virtuelle d'Édimbourg (liens avec 4400 sites de grande qualité en sciences de l'ingénieur)

\begin{tabular}{|c|c|}
\hline Internet PointerGuide (pour le génie civil) & hitp://www.dtv.dk/ipg/4/ \\
\hline Guide des ressources en génie civil sur l'Tnternet & http://www.guideme.com/CivilEngineering.htm \\
\hline Bibliothèque virtuelle de génie civil & http://www.ce.gatech.edu/WWW-CE \\
\hline $\begin{array}{l}\text { Porte d'entrée du génie civil. Plus de } 250 \text { liens } \\
\text { vers les professionnels, chercheurs et enseignants }\end{array}$ & http://www.eudil.fr/eudil/genciv/index.htm \\
\hline $\begin{array}{l}\text { Site documentaire de l'ENPC, nombreux services } \\
\text { et liens en France et en Europe pour le génie civil }\end{array}$ & http://www.enpc.fr/doc/doc.html \\
\hline Bibliothèque virtuelle de géotechnique & http://geotech.civen.okstate.edu/wwwvl/inndex.htm \\
\hline Guide des ressources en géotechnique sur l'Internet & http:/www.guideme.com/Civil/GeotechnicalEngineering,htm \\
\hline $\begin{array}{l}\text { Annuaire de ressources documentaires } \\
\text { et de liens en géotechnique }\end{array}$ & http://www. wheretogeo.com \\
\hline Bibliothèques virtuelles en hydrologie & $\begin{array}{l}\text { http://terrassa.pnl.gov :2080/EESC/resourcelist/hydrology.html } \\
\text { http://www.cleanh2o.com }\end{array}$ \\
\hline Bibliothèque virtuelle pour les sciences de l'environnement & http://earthsystems.org/vl/ \\
\hline Moteur de recherche pour la géotechnique de l'environnement & http://www.geoindex.com \\
\hline $\begin{array}{l}\text { Répertoire des ressources en sciences de la terre } \\
\text { (université de Rennes 1) }\end{array}$ & http://www.geosciences, univ-rennes 1,fr/biblio \\
\hline $\begin{array}{l}\text { Geoscience information center. Moteur de recherche } \\
\text { pour les sciences de la terre }\end{array}$ & http://www.geosciences.org/links \\
\hline $\begin{array}{l}\text { Sélection des « meilleurs x sites pour les géologues } \\
\text { et les sciences de la terre }\end{array}$ & $\begin{array}{l}\text { http://www- } \\
\text { sul.stanford.edu/depts/branner/information/sel.geo.html }\end{array}$ \\
\hline
\end{tabular}

http://www.eevliac.uk/

$\mathrm{http}: / / w w w . d t v, d k / j \mathrm{pg} / 4$

sul.stanford.edu/depts/branner/information/sel.geo.htm/ 


\section{Fureter sur les sites de documentation}

La recherche peut aussi débuter par une visite des sites documentaires gérés par les Écoles, les Universités ou les grands Centres de recherche (tableau V). Ils proposent souvent des moteurs de recherche internes qui permettent d'obtenir rapidement des informations sur leurs produits, leurs publications, leur organisation, leurs prestations, les cours et formations, etc. ou qui orientent vers une sélection d'autres sites ou de bases de données spécialisées (et gratuites).

Sur ces sites gérés par des documentalistes, on trouve également des guides en ligne pour la recherche d'informations sur l'Internet. On conseille notamment la lecture des deux documents suivants: Sentiers d'Accès et Pistes de Recherche d'Informations Scientifiques et Techniques sur l'Internet (projet SAPRISTI de la Documentation de I'INSA de Lyon (Joly et al., 1999)) et Recherche d'Information sur l'Internet (projet RIsI, Lardy, 1999).

TABLEAUV Catalogues des bibliothèques sur l'Internet (tableau établi en octobre 2000). Catalogues of intermet libraries. Table dated October 2000.

Nom de l'éditeur
Adresse sur IThternet:

http://www.enssib.fr/Enssib/t_bibliofr.htm http://www,abf.asso.fr/liens/bibliotheques-france.html http://sunsite.berkeley.edu/Libweb/ http://info.lib.uh.edu/indexes/geosci.htim http://www.shef.ac.uk/-lib/cdlists/cdssub.html

http://www.uottawa.ca/library/subjects/civeng/gencivil-tm.html

\section{5}

\section{Cingler vers les sites des Sociétés savantes}

A l'instar du CFMS, la très grande majorité des sociétés savantes disposent aujourd'hui d'un site sur l'Internet. Celui-ci est généralement composé de deux parties : l'une d'accès libre et l'autre réservée aux membres. Dans la partie publique, les liens externes sont souvent accessibles et permettent d'orienter le début d'une recherche en allant sur d'autres sites d'associations, d'écoles ou d'organismes spécialisés. Par exemple, le site de l'Association universitaire de génie civil (www.iut-egletons, unilim.fr/augc/index.htm) établit des liens avec tous les laboratoires de gênie civil français (universités, écoles d'ingénieur, centres techniques), toutes les formations doctorales et les instituts spécialisés (IUP, IUT).

Le chercheur peut aussi consulter des annuaires d'annuaires, comme par exemple un annuaire des sociétés savantes (http://library.uwaterloo.ca/society). Il existe également d'autres sites de type " annuaire » qui permettent de trouver les adresses de laboratoires de recherche d'après leur localisation et leur centre d'intérêt. Par exemple, le site du ministère de la Recherche (www.recherche.gouv.fr) contient les coordonnées des établissements d'enseignement, des écoles doctorales et des laboratoires de recherche français.

\section{6}

\section{Cheminer dans les bases de données bibliographiques gratuites}

Une recherche bibliographique peut être effectuée soit par une analyse directe à l'aide d'un moteur comme Alta Vista ou Yahoo, soit en partant d'un site documentaire, soit en consultant les serveurs des éditeurs de revues ou des services qui fournissent des documents (tableau VI), soit en explorant les bases de données spécialisées et gratuites. Une liste d'environ 400 bases de données gratuites avec les liens correspondants peut être consultée à l'adresse suivante

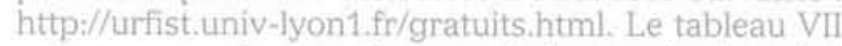
regroupe quelques adresses de bases de données qu'on peut utiliser pour cette approche et qui permettent d'accéder gratuitement aux résumés des références intéressantes. Mais si la lecture et l'impression des résumés sont gratuites, il faudra payer pour obtenir le texte intégral des documents.

Il est également possible de regarder avec profit les sommaires de revues, en passant par une page de liens spécialisés (par exemple : www.tenlinks.com/civil qui établit des liens directs avec 150 journaux et revues) ou de parcourir les journaux électroniques gratuits. Les grandes revues scientifiques possèdent leur propre site et autorisent quelquefois des recherches par mots-clés, voire l'accès aux résumés d'articles.

TABLEAUVI Exemples de bases de données documentaires (consultation gratuite) (tableau établi en octobre 2000). Documentary databases examples (free access). Table dated October 2000.

\begin{tabular}{lll}
\hline Nom & \multicolumn{1}{c}{ Description du contenu } & Adresse sur l'Internet \\
\hline INIST & $\begin{array}{l}10000 \text { périodiques (toute discipline). } 6 \text { millions de références. } \\
\text { Interrogation par mots-clés, Résultat: liste d'articles. }\end{array}$ & http://services.Inist.fr \\
\hline UNCOVER & $\begin{array}{l}18000 \text { périodiques (toute discipline). } 8,8 \text { millions de références depuis 1988. } \\
\text { Interrogation par mots-clês. }\end{array}$ & http://uncweb.carl.org/ \\
\hline
\end{tabular}


TABLEAUVII Exemples de bases de données bibliographiques accessibles gratuitement (interrogation par mots-clés et résumés en ligne) (tableau établi en octobre 2000).

Bibliographic databases examples (free access). Keywords search and online summaries, Table dated October 2000.

\begin{tabular}{|c|c|c|}
\hline Nom & Description du contenu & Adresse sur IInternet \\
\hline ASCE & $\begin{array}{l}80000 \text { références et rẻsumés concernant toutes les publications } \\
\text { de I'ASCE depuls } 1975 \text { (articles, actes de congrès, livres et rapports). }\end{array}$ & http://www.pubs.asce.org/cedbsrch.html \\
\hline NASA & $\begin{array}{l}290000 \text { références et rêsumés dans les domaines de la physique } \\
\text { et géophysique. }\end{array}$ & http://cdsads.u-strasbg.fr/physics_service.html \\
\hline NISEE & $\begin{array}{l}\text { National Information Service for Earthquake Englneering. } \\
80000 \text { références et résumés depuis } 1971 \text {. }\end{array}$ & $\begin{array}{l}\text { http://www.eerc.berkeley,edu/cgi- } \\
\text { bin/texhtmilform=eea }\end{array}$ \\
\hline NTIS & $\begin{array}{l}\text { Banque de rapports des agences de recherche } \\
\text { publiques américaines (plus de } 400000 \text { rapports et leur résumé). }\end{array}$ & http://www.ntis,org/search.htm \\
\hline QUAKELINE & $\begin{array}{l}80000 \text { références et rèsumés dans le domaine du gènie. } \\
\text { parasismique. }\end{array}$ & http://nceer,eng.buffalo.edu/bin/ \\
\hline SG-LINE & $\begin{array}{l}55000 \text { références d'articles et de rapports en gèotechnique } \\
\text { depuis } 1976 \text {. }\end{array}$ & http://www.swedgeo.se/login/signin.asp \\
\hline UWTN & $\begin{array}{l}\text { Universities Water Information Network. } 265000 \text { références } \\
\text { et résumés dans le domaine de l'hydrologie et des problèmes } \\
\text { liès à leau (de } 1967 \text { à octobre } 1993 \text { ). Base gèrêe par le Water } \\
\text { Resources Scientific lnformation Center. }\end{array}$ & http:/www uwin.situedu/databases/wrsic/index.html \\
\hline Cyberthèses & $\begin{array}{l}\text { Base de donnêes de signalement des thèses en ligne. } \\
\text { Beaucoup de thèses en médecine et en sociologie. }\end{array}$ & http://www.cybertheses.org/cybertheses/tramebas $2 . h t m l$ \\
\hline THESA & $\begin{array}{l}\text { Banque de données française des sujets de thèses en cours } \\
\text { dans } 22 \text { Grandes Écoles (environ } 600 \text { théses répertoriées). }\end{array}$ & http://thesa.inist.fr \\
\hline TheseNet & $\begin{array}{l}\text { Catalogue des thèses soutenues en France depuis } 1972 . \\
\text { notamment pour les sciences exactes et appliquées. }\end{array}$ & http: //thesenet abes.fir \\
\hline $\begin{array}{l}\text { Dissertation } \\
\text { Express }\end{array}$ & Répertoire de plus d'un million de thèses américaines. & http://wwwlib.uml.com/dxweb/search \\
\hline ATTIC & Base de données américaine pour la protection de l'environnement. & http://www.epa.gov/attic/ \\
\hline
\end{tabular}

\section{Lire des journaux électroniques}

Plusieurs centaines de magazines et de journaux électroniques (accessibles gratuitement pour la plupart) ont été développées sur l'Internet. Ces sites proposent généralement :

- un ensemble de rubriques d'actualité :

- des articles scientifiques et techniques

- des listes de sites commentés ;

- des pages d'annonces diverses :

- des produits variés (logiciels, technologies, vidéos, etc.).

Par exemple, un des journaux les plus anciens, créé en 1996, est consacré à la géotechnique (http://geotech.civen.okstate.edu/ejge/index.htm). Environ une dizaine d'articles sont ainsi ( publiés » chaque année. Le volume 5 pour l'année 2000 comprend déjà onze articles. Une liste des journaux électroniques disponibles gratuitement peut être obtenue à l'adresse suivante: www.hw.ac.uk/libwww/faculty/genres.html\#ejn.

A côté des journaux électroniques qui sont organisés comme les revues scientifiques sur papier avec un comité de lecture et une présentation uniforme généralement agréable, on trouve des magazines de l'Internet entièrement consacrés à un domaine scientifique, qui fonctionnent comme un hebdomadaire traditionnel avec les petites annonces (conférences, emplois, etc.), le courrier des lecteurs (FAQ, Frequently Asked Questions ou «Foire Aux Questions »), des livres, des cri- tiques, la publicité, le hit-parade des sites les plus visités mais aussi, puisque les nouvelles technologies le permettent, des images, des animations, des vidéos, des logiciels, etc. Un des exemples intéressants est le magazine de la géotechnique sur l'Internet (http://geotech.civen.okstate.edu/magazine).

\section{5}

\section{Comment évaluer l'information obtenue gratuitement}

L'Internet est un outil avec lequel il est rare de revenir bredouille. C'est plutôt le contraire ; on est rapidement noyé par les informations et on a du mal à faire le tri entre le bon, le mauvais et l'inutile. Il est également légitime de se poser des questions sur la validité de ces informations. Si l'accès a été payé, la responsabilité de l'éditeur du site est normalement engagée et on devrait connaître facilement le degré de sérieux des services proposés. En revanche, la gratuité ne donne aucune garantie particulière. Quand il s'agit d'informations bibliographiques, la sanction vient de leur lecture qui permet d'évaluer les travaux présentés. Le seul inconvénient est de perdre du temps et de l'argent pour des photocopies peu intéressantes : mais c'est au fond le risque de toute étude bibliographique à vocation exhaustive.

Pour les autres informations qui n'ont par nature pas été expertisées comme l'est une publication, un regard critique et une évaluation s'imposent. Outre le 
jugement habituel porté sur un travail scientifique, Joly et al. (1999) ont proposé une grille d'évaluation fondèe sur des critères relatifs aux aspects suivants :

- le site. Est-il connu de collègues ou des documentalistes? La manière d'y arriver peut indiquer sa qualité, s'il a été recommandé par un autre site lui-même de bonne réputation. Est-il en construction depuis plusieurs mois? Cela peut suggérer un manque de moyens pour mener à terme le projet. Comment se présente l'environnement? Peut-on se déplacer facilement dans les pages Web? Les enchainements sont-ils logiques? Existe-t-il la possibilité d'effectuer des recherches dans ce site? avec des mots-clés ? des opérateurs booléens?

- le contenu. Les objectifs du site sont-ils clairs? Quelle est son audience (nombre de visiteurs) ? Quel est le degré de sérieux dans la présentation et le style des textes? Les sources sont-elles bien identifiées? Le document inclut-il une bibliographie ? Les règles documentaires sont-elles respectées ? Procède-t-on régulièrement à la mise à jour des informations ? Quelle est la fraicheur de celles-ci? Comment sont gérés les liens thématiques avec d'autres sites? Sont-ils pertinents et validés régulièrement? Une sortie papier est-elle possible? L'information est-elle protégée par un copyright? Qui en détient les droits?

- l'organisme gestionnaire du site. Est-il clairement nommé ? Est-ce une organisation gouvernementale? Une université? Une entreprise? Y a-t-il une affiliation à une Société Savante ? S'il s'agit d'une page personnelle, est-il possible de retourner à la page d'accueil de lorganisme?

- l'auteur. Est-il reconnu dans cette discipline? Quelles sont ses compétences? Sa situation professionnelle? S'il s'agit d'une page personnelle, fournit-il son curriculum vitae? Si l'auteur n'est pas connu, est-il recommandé ou cité par quelqu'un de connu ? S'exprime-t-il en son nom propre ou en celul de son organisme? Peut-on le contacter par son adresse électronique pour obtenir plus de précisions?

- la gratuité. Toutes les informations sont-elles gratuites ou en partie seulement? Sont-elles accompagnées de publicité sur le méme sujet? Les publicités sont-elles clairement séparées du contenu du site? Des intérêts financiers sont-ils en jeu ? Le site présente-t-il des produits ou services vendus par l'auteur ou l'organisme? etc.

Ces questions permettent de se faire une idée sur la sûreté et le sérieux des informations recueillies. II n'y a évidemment aucune garantie, mais on peut se forger une intime conviction sur la validité d'un site. De manière plus prosaïque, des groupes d'experts semblent se mettre en place pour agréer des sites par spécialités et veiller à leur qualité (Joly et al., 1999). Cela se pratique déjà en économie, en management et bien sûr en médecine.

\section{6}

\section{Conclusion}

Les trois comités français : de géologie de l'ingénieur (CFGI), de mécanique des roches (CFMR), de mécanique des sols et de géotechnique (CFMS) et la Revue Française de Géotechnique (RFG) ont mis en service un site commun sur l'Internet depuis mai 2000 (www.geotechnique.org). Ce site permet d'accéder aux pages propres à chaque comité et à celle de la RFG.

Pour sa part, le CFMS a développé ses pages avec pour objectifs principaux de faire connaitre ses activités techniques, d'être un lien pour les géotechniciens francophones et une « porte d'entrée » pour des recherches sur le Web. Ses différents services sont offerts aux membres de l'association et à tous les internautes du monde.

Cet article avait aussi pour but de montrer l'intérêt de l'Internet aux géotechniciens réticents et de prodiguer quelques conseils pour ceux qui aimeraient tenter l'aventure de la navigation en solitaire mais qui se sentent peu familiarisés avec ce nouveau moyen de communication. Les potentialités de l'Internet sont immenses en termes de communication, de recherche rapide d'informations, de sources documentaires et d'établissement de contact entre personnes d'une mème communauté d'intérêt. Si le site du CFMS peut être vu comme un outil capable de rendre des services aux géotechniciens, nous aurons atteint notre but.

\section{Bibliographie}

Joly M.. Mermet J.-M., Bion N.. Burlat J.M., Pradal T., Prud'homme B. (1999) u Sentiers d'accès et pistes de recherche d'informations scientifiques et techniques sur I'Internet. Projet SAPRISTI $p$. Doc'INSA, INSA de Lyon. (Document accessible à l'adresse suivante: http://csidoc,insa-lyon.fr/sapristi/digest. html).

Lardy J.-P. (1999) - \& Recherche dinformation sur IInternet. Outils et méthodes. Projet Rlsl n. Lyon. (Document accessible à l'adresse suivante: http://www.adbs, fr/acibs/viepro/sinfoint/ardy/risi.htm).
Le Roux A.. Mestat P. Lacour P. (2000) "Sources documentaires sur l'Internet: quelques conseils pour obtenir des informations en génie civil 10 . Bulletin des Laboratoires des Ponts et Chaussées, $n^{\circ} 229$, p. 83-96. 